\title{
Indicadores de depressão e de declínio cognitivo em idosos institucionalizados: um estudo de caso
}

\author{
Edvaldo Soares", Stella Maira Demartini" \\ Sebastião Marcos Ribeiro de Carvalho"*
}

\section{Resumo}

Este estudo de caso objetivou investigar a prevalência de sintomatologia para depressão (DP) e declínio cognitivo (DC) em idosos institucionalizados; verificar a existência de correlações entre DP e DC com idade, sexo e escolaridade; e, analisar as possíveis correlações entre sintomatologia para DP e DC. Para a realização da pesquisa, foram selecionados, em duas instituições de longa permanência para idosos (ILPIs), 24 sujeitos, os quais foram classificados quanto ao sexo, à escolaridade e à faixa etária. Para verificar a ocorrência de DC, foi aplicado o Mini-Exame do Estado Mental (MEEM) e, para a incidência de DP, a Escala de Depressão Geriátrica (EDG). Os dados foram tabulados e analisados descritivamente. Para o estudo das relações entre variáveis quantitativas, utilizou-se o teste de correlação de Pearson e, quando necessário, o de Spearmann; para a comparação entre dois grupos independentes, foi utilizado o teste t de Student; para a verificação da associação entre sexo, escolaridade, in- dicativo de DP e de DC, foi utilizado o teste $\chi^{2}$ de Pearson e, por restrição teórica, o teste exato de Fisher. Adotou-se o nível de 5\% de probabilidade para a rejeição da hipótese de nulidade em todos os testes. Os resultados demonstraram que $50 \%$ dos sujeitos da pesquisa apresentaram indicativo de DP e, $54,2 \%$, de DC. Não foram observadas associações entre: sexo e DP ( $p=0,414)$; sexo e DC ( $p=0,219)$; escolaridade e DC $(p=0,527)$. Verificou-se correlação regular positiva entre idade e DP $(r=0,557 ; p=$ $0,005)$ e correlação regular negativa entre DP e DC $(r=-0,406 ; p=0,049)$. Os dados sugerem que a DP pode ser uma reação ao DC percebido pelo sujeito. Porém não podem ser descartadas hipóteses que indicam que a DP é um fator de risco para o DC e para diagnóstico de demência (DM), o que sugere a importância do monitoramento e do tratamento de episódios depressivos em populações idosas.

Palavras-chave: Idosos. Instituição geriátrica. Depressão. Declínio cognitivo.

* Doutor em Neurociência e Comportamento pela Universidade de São Paulo. Docente do Departamento de Psicologia da Educação da Universidade Estadual de São Paulo - Campus de Marília-SP. Coordenador do Grupo de Pesquisa em Neurociências e Comportamento: Memória, Plasticidade, Envelhecimento e Qualidade de Vida. E-mail: edsoares@marilia.unesp.br. Endereço para correspondência: Avenida Hygino Muzzi Filho, 737. CEP 17500-925, Marília-SP.

** Terapeuta Ocupacional. Grupo de Pesquisa em Neurociências e Comportamento: Memória, Plasticidade, Envelhecimento e Qualidade de Vida - Faculdade de Filosofia e Ciências - Universidade Estadual de São Paulo. E-mail: stellademartini@hotmail.com

*** Doutor em Energia na Agricultura e meste em Medicina Interna e Terapêutica pela UNIFESP. Docente do Departamento de Psicologia da Educação e pesquisador do Laboratório de Neurociência Cognitiva - LaNeC, Universidade Estadual Paulista - Unesp - Campus de Marília SP.

$\rightarrow$ http://dx.doi.org/10.5335/rbceh.2013.1727 


\section{Introdução}

Conforme o DSM-IV-TR, depressão (DP) é um transtorno de humor que se caracteriza pela presença de alguns dos seguintes sintomas: 1) estado deprimido (sentir-se deprimido a maior parte do tempo); 2) anedonia (interesse diminuído ou perda de prazer para realizar as atividades de rotina); 3) Sensação de inutilidade ou culpa excessiva; 4) dificuldade de concentração (habilidade frequentemente diminuída para pensar e concentrar-se); 5) fadiga ou perda de energia; 6) distúrbios do sono (insônia ou hipersônia praticamente diárias); 7) problemas psicomotores (agitação ou retardo psicomotor); 8) perda ou ganho significativo de peso, na ausência de regime alimentar; 9) Ideias recorrentes de morte ou suicídio. Conforme o número de sintomas apresentados, a DP pode, segundo o DSM-IV-TR, ser classificada em três categorias: 1) depressão menor (de dois a quatro sintomas por duas ou mais semanas, incluindo estado deprimido ou anedonia); 2) distimia (três ou quatro sintomas, incluindo estado deprimido, durante dois anos, no mínimo) e, 3) depressão maior (cinco ou mais sintomas por duas semanas ou mais, incluindo obrigatoriamente estado deprimido ou anedonia) (APA, 2008).

As taxas de prevalência de depressão entre populações idosas estão entre 10,3\% e $13,5 \%$ na população mundial e de até $15 \%$ na população brasileira. (REYS et al., 2006; HEISER, 2004). Pesquisas realizadas no Brasil, na década de 1990, sugerem que a prevalência de transtornos mentais, dentre os quais figura a depressão, aumenta com a idade. (LIMA et al., 1999; LIMA et al., 1996; MARI et al., 1993). Vários estudos, apesar de ressalvas quanto à generalização, têm indicado uma prevalência maior de depressão em mulheres do que em homens (KOCKLER; HEUN, 2002; ANGST et al., 2002). No que se refere à relação entre escolaridade e DP, as pesquisas ainda são inconclusivas. Por exemplo, Cacciatore e colaboradores (1998) indicaram que quanto menor a escolaridade, maior a incidência de DP. Entretanto algumas pesquisas concebem que o grau de escolaridade apresentava fraca associação com DP (PAULO; YASSUDA, 2010; BLACK; MARKIDES; MILLER, 1998).

A cognição envolve habilidades de pensar, perceber, lembrar, sentir, raciocinar e responder aos estímulos externos (SOARES; COELHO; CARVALHO, 2012; RABELO, 2009). O declínio cognitivo, especialmente no que se refere à memória, é um evento normal do envelhecimento, podendo inclusive se manifestar antes do 40 anos de idade (SWEATT, 2010). Indivíduos idosos apresentam grande variabilidade intra e interindividual em termos de funcionamento cognitivo, o que explica o fato de, em populações idosas, existir uma variabilidade em termos de declínio em diferentes funções cognitivas e, até mesmo, o aperfeiçoamento em outras funções. (STÜSSI; FORTES; RABELO, 2010; RABELO, 2009). DC é uma característica universal nos indivíduos que apresentam diagnóstico de demência (APA, 2008) e seu quadro é caracterizado por dificuldades cognitivas, especialmente de memória (SOARES; COELHO; CARVALHO, 2012). Bruscoli e Lovestone (2004) destacam que a taxa de conversão de DC para a doença de Alzheimer (DA) é 
de $10 \%$ a $15 \%$ ao ano, enquanto pessoas saudáveis apresentam taxa de $1 \%$ a $2 \%$ ao ano. Almeida (1998) sugere que tanto idade quanto educação tem efeitos significativos sobre a cognição. Nessa mesma perspectiva, Diniz, Volpe e Tavares (2007) indicam que tanto a idade quanto o nível educacional produzem influências estatisticamente significantes em relação à capacidade cognitiva. Resultados relativos à prevalência de $\mathrm{DC}$ em relação ao sexo ainda não são conclusivos, considerando que pesquisas realizadas em vários países mostram resultados divergentes (DINIZ; VOLPE; TAVARES, 2007; HAN et al., 2007; ANDERSON et al., 2007; NITRINI et al., 2004).

DP e DC são comuns em adultos mais velhos. A relação entre tais transtornos é bi-direcional e complexa (GANGULI, 2009) e tem sido interpretada de forma diversa. Jorm (2000) considera que não há evidências suficientes para afirmar que a DP é um fator de risco para DM ou DC. Entretanto, pesquisas indicam, já há algum tempo, que sintomas de DP apresentam-se frequentemente como forma de apresentação de um transtorno cognitivo, como na DA (LYKETSOS et al., 1997; EDWARDS; LINDQUIST; YAFFE, 2004). Da mesma forma, não é raro o DC como alteração secundária à DP (RAPP et al., 2005). Pesquisas têm estimado que a prevalência de $\mathrm{DC}$ em indivíduos com DP está entre $25 \%$ e $50 \%$ dos casos, ao passo que a prevalência de DC sem DP é de $3 \%$ a $6 \%$ (LEE et al., 2007; GANGULI et al., 2004). Reischies e Neu (2000) destacam que o DC durante um episódio de depressão pode persistir mesmo após a melhora dos sintomas de
DP. Há uma tendência na literatura especializada em indicar a DP como fator de risco para DA (OWNBY et al., 2006). Seguindo a mesma tendência, estudos longitudinais sugerem que a DP pode preceder o desenvolvimento de DM, ou mesmo constituir um fator de risco para 0 aparecimento de DA. (ALEXOPOULOS et al., 2002; YOCHIM, 2006). Geerlings e colaboradores (2000) associam a DP ao aumento do risco de DA e de DC, respectivamente, mas apenas nos indivíduos com níveis mais elevados de educação. Segundo esses estudos, em um subgrupo de idosos mais escolarizados, a DP pode ser uma manifestação precoce de DA, mesmo antes dos sintomas relacionados aos deficit cognitivos tornarem-se aparentes. (GEERLINGS; BOUTER; SCHOEVERS et al., 2000).

Sullivan e demais autores (2013), em estudo acerca da associação entre DP e DC acelerado em pacientes com diabetes tipo 2 , indicaram que os sujeitos com indicativo de DP apresentavam maior DC. Para Stewart (2004), a DP é um fator de risco para DC e para DM, sendo forte a hipótese de que a DP pode acelerar ou mesmo desencadear processos neurodegenerativos. Saczynski et al. (2010) concluem que a DP está associada a um aumento do risco de DM, especialmente de DA. Gatz, St. John e Montegomery (2005) indicaram que a ocorrência de sintomas depressivos em idosos, por mais de cinco anos, pode prever o aparecimento de DM, especialmente a DA. Em função disso, esses pesquisadores afirmam a necessidade de monitoramento cognitivo de pacientes mais velhos com sintomas de DP. Entretanto, é importante destacar que a incidência de DP diminui a 
validade preditiva das queixas de memória quanto ao diagnóstico de DM. (JORM et al., 2001).

Perrino e colaboradores (2008) sugerem que o DC pode prever sintomas depressivos em adultos mais velhos. Alguns estudos têm sugerido que, entre idosos, as queixas subjetivas em termos de DC, especialmente de memória, não são apenas secundárias à DP, mas expressam, em parte, uma autopercepção realística de DC. Ou seja, a percepção subjetiva de DC, especialmente de deficit de memória, estaria mais associada à $\mathrm{DP}$ (GUERREIRO et al., 2006; WANG et al., 2004; XAVIER et al., 2001; WANG et al., 2000). Porém, pode-se ignorar o fato de que idosos com DC podem melhorar seu desempenho em tarefas que envolvam memória e funções executivas a partir de tratamento psicofarmacológico. Alguns indivíduos que apresentam DP mais tardiamente não alcançam níveis normais em tais tarefas. Esse subgrupo de idosos seria mais propenso ao desenvolvimento de DM. (ALEXOPOULOS et al., 2000; BUTTERS et al., 2000).

De forma geral, conforme Stewart (2004), em relação à associação entre DP e DC, podemos destacar três hipóteses possíveis: 1) a DP pode ser simplesmente uma reação a uma deterioração percebida pelo sujeito, na sua função cognitiva; 2) a função cognitiva pode ser prejudicada durante um episódio depressivo em função da redução da atenção e da motivação e, 3) a DP é um sintoma de DM e não um fator de risco. De acordo com Wilson, Mendes De Leon e Bennett (2004) essas hipóteses são limitadas. Tais limitações, de acordo com Stewart (2004), ocorrem porque, no curso da vida, diversos fatores afetam o estado de humor, fazendo-o variar. Existem, segundo o autor, relações interdependentes entre função cognitiva e outros aspectos da saúde, como por exemplo, doenças vasculares (STEWART, 2004). Esse fato por si só dificulta a possibilidade de testagem de hipóteses e o estabelecimento de relações de causa-efeito. Entretanto, a análise de conjunto de dados longitudinais; invariavelmente limitados em função do grande número de variáveis a serem analisadas; podem contribuir substancialmente para esse campo de pesquisa. Apesar das diferentes interpretações da relação entre DP - DC, a hipótese de que a DP pode acelerar ou mesmo desencadear processos neurodegenerativos é a mais forte na atualidade. Tal fato é interessante, pois, conforme Stewart (2004) indica implicitamente a possibilidade de prevenção de DC e de DM.

Considerando a hipótese de que a DP é um dos fatores de risco importantes no sentido de desencadear ou acelerar o DC, este estudo objetivou investigar a prevalência de sintomatologia para DP e DC em idosos institucionalizados; verificar a existência de correlações entre DP e DC com idade, sexo e escolaridade; e, analisar as possíveis correlações entre sintomatologia para DP e DC.

Dados levantados pelo Laboratório de Neurociência Cognitiva ( $\mathrm{LaNeC}$ ), da Universidade Estadual Paulista - Campus de Marília-SP, têm indicado alta incidência de sintomatologia para DP e de DC em idosos residentes. Tais observações apontam para a hipótese de que o processo de institucionalização, por ser um evento altamente estressante, induz 
à $\mathrm{DP}$ e ao $\mathrm{DC}$ e, consequentemente, à piora no estado geral de saúde (SOARES et al., 2010; 2012). Entretanto, dessa hipótese decorrem três questões importantes: 1) No contexto da institucionalização, existiriam correlações entre DP e DC com fatores tais como idade, sexo e escolaridade? 2) No contexto de institucionalização, haveria algum tipo de correlação entre DP e DC? 3) Qual ou quais hipóteses poderiam explicar a ocorrência ou não de correlação entre DP e DC?

\section{Métodos e procedimentos}

Para este estudo de caso, foram selecionadas duas ILPIs localizadas na cidade de Marília-SP. A ILPI 1 contava com uma população de 47 idosos residentes , dos quais $57 \%$ eram do sexo masculino e $43 \%$ feminino, enquanto a ILPI'2 contava com 49 idosos, dos quais $45 \%$ do sexo masculino e, $55 \%$ feminino. Em termos de faixa etária, na ILPI1, a idade média é de 70,8 anos e, na ILPI2 é de 71,4 anos. Foram sujeitos da pesquisa 24 idosos residentes (12 ILPI1 e 12 ILPI2), de ambos os sexos, com idade entre 59 e 89 anos, selecionados conforme critérios de elegibilidade estabelecidos, sendo incluídos apenas os sujeitos que aceitassem participar da pesquisa e, entre esses, os que apresentassem capacidades auditiva, de compreensão linguística e cognitiva suficientes para compreender e responder os instrumentos de coleta de dados. A amostra foi distribuída, conforme suas características, dentro dos seguintes grupos/subgrupos: a) sexo (feminino, $\mathrm{n}=12$; masculino, $\mathrm{n}=12$ ); $\mathrm{b}$ ) escolaridade (escolarizados, $\mathrm{n}=14$; não escolarizados, $\mathrm{n}=10$ ); e, c) faixa etária $(59-64, \mathrm{n}=4 ; 65-70, \mathrm{n}=4 ; 71-76, \mathrm{n}=6$;
77-83, $\mathrm{n}=7 ; \mathrm{e}, 84-89, \mathrm{n}=3$ ). Para verificar a ocorrência de DC, foi aplicado o Mini-Exame do Estado Mental (MEEM) e, para a incidência de DP, a Escala de Depressão Geriátrica (EDG).

O MEEM, elaborado por Folstein e colaboradores (1975), é composto por uma avaliação objetiva da orientação, atenção, concentração, memória, cálculo e linguagem. O escore máximo possível é de 30 pontos. Nesta pesquisa, seguindo as orientações de Almeida (1998), foram utilizados pontos de corte diferenciados conforme a escolaridade dos sujeitos. Entre idosos com algum grau de escolaridade, foi utilizado o ponto de corte 24 (0-24 = indicativo de declínio cognitivo; 25 ou mais $=$ normal) $\mathrm{e}$, para os sujeitos sem escolaridade, o ponto de corte 19 (019 = indicativo de declínio cognitivo; 20 ou mais = normal). A EDG, desenvolvida em língua inglesa, por Yesavage et al. (1983) constitui um instrumento largamente empregado para rastrear sintomas depressivos em populações idosas, inclusive de idosos institucionalizados (KOEHLER; RABINOWITZ; HIRDES, 2005; MOSSEY; GALLAGHER, 2004). A escala original é composta por 30 perguntas (negativas/afirmativas), que evitam a esfera das queixas somáticas. O escore total é dado pela somatória das respostas indicativas de depressão. Para a EDG-30, adotou-se o ponto de corte de $11(0-10=$ sem suspeita de DP e 11 ou mais = com suspeita de DP) (SOUSA et al., 2007).

Os dados foram tabulados e analisados descritivamente. Para o estudo das relações entre variáveis quantitativas, utilizou-se o teste de correlação de Pearson; para a comparação entre dois grupos independentes, foi utilizado o teste $\mathrm{t}$ de Student; para a verificação 
da associação entre sexo, escolaridade, indicativo de DP e de DC, foi utilizado o teste $\chi^{2}$ de Pearson; $e$, por restrição teórica, o teste exato de Fisher (T.E.F.). Para a avaliação qualitativa do coeficiente de correlação de Pearson (r) e, quando necessário, por restrição teórica, o de Spearmann. Quanto à intensidade, foram considerados os seguintes graus de correlação (r), conforme proposto por Callegari-Jacques (2003): 0 (nula); 0,0 I- 0,3 (fraca); 0,3 ।- 0,6 (regular); 0,6 I- 0,9 (forte); 0,9 |- 1,0 (muito forte) e, 1 (plena ou perfeita). Foi adotado o nível de significância de $5 \%$ de probabilidade para a rejeição da hipótese nula em todos os testes (ARMITAGE; BERRY, 1994). A pesquisa seguiu todos os dispositivos éticos indicados na resolução 196/96, tendo sido aprovada pelo Comitê de Ética na Pesquisa da Faculdade de Filosofia e Ciências (FFC) da Universidade Estadual Paulista (UNESP), Campus de Marília-SP (Parecer 1.198/2010).

\section{Resultados e discussão}

Conforme indicado, a amostra selecionada para este estudo foi de 24 sujeitos, sendo $50 \%$ do sexo masculino e $50 \%$ do feminino. Desse total, $41,7 \%$ dos sujeitos concentravam-se na faixa etária de 77 a 89 anos. Em termos de faixa etária, a média geral foi de 74,5 anos $(\mathrm{dp}=$ 8,5), sendo, na ILPI'1, a média de 76,0 $\operatorname{anos}(d p=9,3)$, enquanto que, na ILPI'2, de $72,9(\mathrm{dp}=7,8)$.

A partir da aplicação da EDG, foi constatado que 12 sujeitos $(50 \%$ da amostra) apresentavam indicativo de $\mathrm{DP}$, dentre os quais $5(41,7,8 \%)$ eram do sexo feminino e $7(58,2 \%)$ do masculino. Não foi observada, em relação aos sexos, diferença significativa entre a pontuação média obtida na EDG ( $\mathrm{p}>0,05)$ (masculino $=10,1[\mathrm{dp}=5,3]$ e feminino $=10,3$ $[\mathrm{dp}=6,9]$ ), o que também se mostra verdadeiro com relação ao indicativo de DP na amostra analisada $(\mathrm{p}=0,414)$. Porém, os números absolutos contrariam dados da literatura que apontam para uma prevalência maior de DP entre mulheres do que em homens (KOCKLER; HEUN, 2002; ANGST et al.; 2002) e são coerentes com dados que indicam que pelo menos 50\% dos idosos institucionalizados apresentam episódios depressivos (HEISER, 2004).

Em relação à faixa etária, foi observado que, entre os sujeitos do sexo masculino que apresentaram indicativo de DP $(29,2 \%), 4,2 \%$ estavam na faixa etária entre 59-64 anos; 8,3\% entre 71-76 anos; $12,5 \%$ entre 77 e 83 anos, e, $4,2 \%$ entre 84 e 89 anos. Dos sujeitos do sexo feminino que apresentaram indicativo de DP $(21,0 \%), 8,3 \%$ estavam na faixa etária entre os 71-76 anos; $4,2 \%$ entre os 77-83 e 8,3\% entre os 84 e 89 anos. Em ambos os sexos, nenhum sujeito na faixa etária entre os 65 e 70 anos de idade apresentou indicativo de DP.

Os resultados do estudo da relação entre idade e DP apontaram, para esta amostra, uma correlação regular positiva $\left(r_{\text {Pearson }}=0,557 ; \mathrm{p}=0,005\right)$, o que corrobora os achados da literatura para populações brasileiras (LIMA et al., 1999; MARI et al., 1993).

Em relação à escolaridade, entre os que apresentaram indicativo de DP, a maioria era de escolarizados $(58,3 \%)$. O resultado do teste exato de Fisher (T.E.F) (Tabela 1) indica independência $(\mathrm{p}=$ $1,000)$ entre escolaridade e sexo. 
Tabela 1. Distribuição dos sujeitos com indicativo de DP quanto à escolaridade por sexo

\begin{tabular}{lcccc}
\hline & \multicolumn{2}{c}{ Escolaridade } & \multicolumn{2}{c}{ Não escolarizados } \\
\hline Sexo & Feminino & Masculino & Feminino & Masculino \\
$\%$ & $33,3(4)$ & $25,0(3)$ & $16,7(2)$ & $25,0(3)$ \\
$\%$ Total & \multicolumn{2}{c}{$58,3(7)$} & & $41,7(5)$ \\
T.E.F: $p=1,000$ & & & \\
\hline
\end{tabular}

Em relação ao DC, na amostra analisada, foi constatado que $13(54,2 \%)$ sujeitos apresentam indicativos de DC. Desse total, $61,6 \%$ (8) eram do sexo feminino e $38,4 \%$ do sexo masculino. A partir da aplicação do T.E.F, não se observou associação entre escolaridade e DC ( $\mathrm{p}=$ 0,527 ) (Tabela 2). Entretanto, os números absolutos indicam uma prevalência maior de sujeitos com indicativo de DC entre os não escolarizados $(61,5 \%)$ do que entre os escolarizados (38,5\%). Esses dados são coerentes com os de Almeida (1998) no que se refere à relação entre os resultados obtidos no MEEM e a escolaridade. Também não foi identificada relação entre sexo e DC $(\mathrm{p}=0,565)$.

Tabela 2. Distribuição dos sujeitos com indicativo e sem indicativo de DC quanto à escolaridade

\begin{tabular}{lcc}
\hline \multicolumn{1}{c}{ Escolaridade } & Indicativo de declínio cognitivo & Sem indicativo de declínio cognitivo \\
\hline$\%$ Escolarizados & $38,5(5)$ & $45,5(5)$ \\
$\%$ Não escolarizados & $61,5(8)$ & $54,5(6)$ \\
$\%$ Total & $100(13)$ & $100(11)$ \\
T.E.F: $p=0,527$ & & \\
\hline
\end{tabular}

No que se refere à faixa etária, observou-se que, dos sujeitos que apresentaram indicativo de $\mathrm{DC}, 50,0 \%$ concentravam-se na faixa etária entre 71 e 89 anos. Apesar dos resultados apontarem, nesta amostra, para uma correlação negativa, porém, não significativa $(\mathrm{r}=-0,228$; $\mathrm{p}=0,284$ ), entre DC e faixa etária, não se pode descartar, conforme vem sendo apontado na literatura, o impacto da idade sobre os resultados obtidos no MEEM (ALMEIDA, 1998).
Importante destacar ainda que, do total da amostra, oito sujeitos $(33,3 \%)$ - cinco $(20,8 \%)$ do sexo feminino e três $(12,5 \%)$ do masculino - apresentaram concomitantemente indicativo de DP e de DC. Em relação à faixa etária, $4,2 \%$ dos indivíduos com idade entre 59-64 anos; $12,5 \%$ entre $71-76$ anos; $8,3 \%$ entre 77-83 anos e, $8,3 \%$ dos sujeitos com idade entre 84-89 anos apresentaram indicativo de DP e de DC. No que se refere à escolaridade, $25 \%$ dos sujeitos sem 
escolaridade apresentaram indicativo de DP e de DC contra $8,33 \%$ sem qualquer grau de instrução.

A partir dos resultados relativos à incidência de DP e/ou DC, os sujeitos foram classificados em quatro grupos distribuídos do seguinte modo: 1) Sujeitos com indicativo de DP e DC $(33,3 \%)$; 2) Sujeitos sem indicativo de DP e com indicativo de DC (21,0\%); 3) Sujeitos com indicativo de DP e sem indicativo de DC $(4,2 \%)$; e, 4) Sujeitos sem indicativo de $\mathrm{DP}$ e sem indicativo de DC $(41,6 \%)$. O resultado do teste do $\chi^{2}$ para as proporções de indivíduos nos grupos foi não significante $\left(\mathrm{p}_{\text {Monte Carlo }}=0,053\right)$, indicando que essas proporções, relativas à presença de DP e/ou DC, foram similares.

A incidência de DP associada à DC em $33,3 \%$ da amostra confirma dados da literatura que indicam uma prevalência de $25 \%$ a $50 \%$ de DC em indivíduos com DP. Os resultados deste estudo apontam ainda para a ocorrência de correlação significante entre as variáveis EDG e MEEM; ou seja, indicou regular correlação negativa entre EDG e MEEM ( $\mathrm{r}=$ $-0,406 ; p=0,049$ ). Portanto, os dados demonstram que os escores EDG diminuem quando há um aumento nos escores do MEEM, o que sugere que indivíduos com menor indicativo de DC apresentam menor sintomatologia para DP.

A relação entre DP e DC tem se mostrado bi-direcional e complexa (GANGULI, 2009). Os resultados dessa pesquisa reforçam a hipótese apresentada em várias pesquisas de que a DP pode ser uma reação ao DC percebido pelo sujeito (GUERREIRO et al., 2006; WANG et al., 2004; XAVIER et al., 2001; WANG et al.,
2000), bem como não contraria o estudo de Perrino e colaboradores (2008), que indica que o DC pode prever sintomas depressivos, ou mesmo pesquisas que sugerem que, mesmo antes do aparecimento de sintomas relacionados ao DC, a DP pode ser uma manifestação precoce de DA (GEERLINGS; BOUTER; SCHOEVERS et al., 2000).

\section{Considerações finais}

Este estudo de caso indicou, na amostra composta por idosos institucionalizados, significativa incidência de DP e DC. Não foram identificadas relações estatisticamente significativas entre DP e sexo; DC e sexo e entre escolaridade e DC. Os resultados indicaram que, com o aumento da idade, ocorre maior incidência de DC. Entretanto, tal correlação não se mostrou significativa em termos estatísticos. Os dados também sugerem que a DP pode estar associada ao processo de envelhecimento e ainda que, quanto maior o DC, maior a sintomatologia para DP, favorecendo, assim, as hipóteses de que a DP pode ser uma reação ao DC percebido pelo sujeito. Apesar desses indicativos, não podem ser descartadas hipóteses que indicam que a DP é um fator de risco para o DC e para DM ou mesmo a de que a DP é uma manifestação precoce de sintoma de DM. Entretanto, independentemente da hipótese reforçada nesta pesquisa, há que se destacar a importância do monitoramento e do tratamento de episódios depressivos em populações idosas. 


\section{Depression and cognitive decline indicators on institutionalized elderly's: a case study}

This case study objectified investigating the symptomatology prevalence for depression (DP) and cognitive decline (CD) on institutionalized elderly's; verifying the existence of correlations between DP and CD with age, gender and scholarity; and analyzing the possible correlations between symptomatology for DP and CD. For the realization of this research were selected, in two Elderly's Long-Permanence Institutions (ELPIs), 24 subjects, which were classified according to gender, scholarity and age group. To verify the occurrence of CD, the Mini Mental State Examination (MMSE) was applied and, for the incidence of DP, the Geriatric Depression Scale (GDS) was applied. The data were tabulated and descriptively analyzed. For the study of the relations between quantitative variables, the Pearson correlation test was utilized and also the Spearmann test when necessary; for the comparison between two independent groups, the Student's t-test was utilized; for the verification of the association between gender, scholarity, DP and CD indicatives, the Pearson's X2 test and, due to theoretical restriction, the Fisher's exact test were utilized. The level of $5 \%$ probability was adopted for the rejection of nullity's hypothesis on all the tests. The research demonstrated that $50 \%$ of the research' subjects presented DP indicatives and $54.2 \%$ presented CD indicatives. Associations between: gender and DP ( $p=$ $0.414)$, gender and CD $(p=0.219)$, scholarity and CD ( $p=0.527)$ were not observed. Positive regular correlation was verified between age and DP $(r=0.557 ; p=0.005)$ and negative regular correlation was verified between DP and CD $(r=-0.406 ; p=$ 0.049). The data suggest that DP might be a reaction to $C D$ perceived by the subject. However, the hypothesis that indicates DP as a risk factor for CD and DM cannot be overruled, which suggests the importance of monitoring and treating depressive episodes on elderly populations.

Keywords: Elderly. Geriatric institution. Depression. Cognitive decline.

\section{Referências}

ALMEIDA, O. P. Mini-exame do estado mental e o diagnóstico de demência no Brasil. Arquivos de Neuropsiquiatria, São Paulo, v. 56, n. 3B, p. 605-612, 1998.

ALEXOPOULOS, G. S.; BUCKWALTER, K.; OLIN, J.; MARTINEZ, R. et al. Comorbidity of late life depression: an opportunity for research on mechanisms and treatment. Biological Psychiatry, n. 52, p. 543-558, 2002.

ALEXOPOULOS, G. S.; MYERS, B. S.; YOUNG, R. C.; KALAYAM, B. et al. Executive dysfunction and long-term outcomes of geriatric depression.Archives of General Psychiatry, n. 57, p. 285-90, 2000.

ANDERSON, T. M.; BRODATY, H.; TROLLOR, J. N.; ANDREWS, G. Effects of sociodemographic and health variables on Mini-Mental State Exam scores in older Australians. American Journal of the Geriatric Psychiatry, n. 15, p. 467-76, 2007.

ANGST, J.; GAMMA, A.; GASTPAR, M.; et al. Gender differences in depression: epidemiological findings from the European Depres I and II studies. European Archives of Psychiatry and Clinical Neuroscience, v. 252, n. 5, p. 201-209, 2002.

APA - American Psychiatric Association. $M a-$ nual diagnóstico e estatístico de transtornos mentais-DSM-IV-TR ${ }^{T M}$. 4. ed. Porto Alegre: Artmed, 2008.

ARMITAGE, P.; BERRY, G. Statistical methods in medical research. 13. ed. Oxford: Blackwell Scientific, 1994.

BLACK, S. A.; MARKIDES, K. S.; MILLER, T. Q. Correlates of depressive symptomatolo- 
gy among older community-dwelling Mexican Americans: the Hispanic EPESE. The Journals of Gerontology Series B: Psychological Sciences and Social Sciences, v. 53, n. 4, p. S198-S208, 1998.

BRUSCOLI, M.; LOVESTONE, S. I. MCI really just early dementia?A systematic review of conversion studies.InternationalPsychogeriatrics, v. 16, n. 2, p. 129-140, 2004.

BUTTERS, M. A.; BECKER, J. T.; NEBES, R.D.; et al. Changes in cognitive functioning following treatment of late-life depression. American Journal of Psychiatry, n. 157, p. 1949-1954, 2000.

CACCIATORE, F.; GALLO, C.; FERRARA, N. et al. Morbidity patterns in aged population in southern Italy. A survey sampling. Archives of gerontology and geriatrics, v. 26, n. 3, p. 201-213, 1998.

CALLEGARI-JACQUES, S. M. Testes nãoparamétricos. In: Bioestatística: princípios e aplicações; p. 165-184. Porto Alegre: Artmed, 2003, p. 165-184.

DINIZ, B. S. O.; VOLPE, F. M.; TAVARES, A. R. Educational level and age and the performance on the Mini-Mental State Examination in community-dwelling elderly. Revista de Psiquiatria Clínica, São Paulo, v. 34, n. 1, p. 13-17, 2007.

EDWARDS, E. R.; LINDQUIST, K.; YAFFE, K. Clinical profile and course of cognitively normal patients evaluated in memory disorders clinics. Neurology, n. 62, p. 1639-1642, 2004.

FOLSTEIN, M. F., FOLSTEIN, S. E.; MCHUGH, P. R. Mini-mental state: a practical method for grading the cognitive state of patients for the clinician. Journal of Psychiatric Research, n. 12, p. 189-198, 1975.

FORTES, L. H. S. D.; RABELO, D. F. Fatores associados ao declínio cognitivo de idosos residentes na comunidade de Patos de Minas-MG. Revista do Núcleo Interdisciplinar de Pesquisa e Extensão do UNIPAM, Patos de Minas, v. 1, n. 7, p. 53-62, 2010.
GANGULI, M.; DODGE, H. H.; SHEN, C.; DEKOSKY, S. T. Mild cognitive impairment, amnestic type: an epidemiologic study. Neurology, v. 63, p. 115-121, 2004.

GANGULI, M. Depression, cognitive impairment and dementia. Why should clinicians care about the web of causation? Indian Journal of Psychiatry, v. 51, (Suppl. 1), p. S29-S34, 2009.

GATZ, J. L.; TYAS, S. T.; ST. JOHN, P.; MONTEGOMERY, P. Do Depressive Symptoms Predict Alzheimer's Disease and Dementia? Journals of Geronotlogy, Serie A, v. 60, n. 6, p. 744-747, 2005.

GEERLINGS, M. I.; BOUTER, L. M.; SCHOEVERS, R. et al. Depression and risk of cognitive decline and Alzheimer's disease :results of two prospective communitybased studies in The Netherlands. British Journalof Psychiatry, n. 176, p. 568-575, 2000.

GUERREIRO, T. C.; VERAS, S. R.; MOTTA, L. B.; VERONESI, A.; et al. Queixa de memória e disfunção objetiva em idosos que ingressam na oficina da memória UnaTI/ UERJ. Revista Brasileira de Geriatria e Gerontologia, Rio de Janeiro, v. 9, n. 1, p. 7-20, 2006.

HAN, C.; JO, S. A.; JO, I.; KIM, E. et al. An adaptation of the Korean mini-mental state examination (K-MMSE) in elderly Koreans: demographic influence and population-based norms (the AGE study). Archives of Gerontology and Geriatrics, n. 47, p. 302-310, 2007.

HEISER, D. Depression identification in the long-term care setting: the GDS vs. the MDS. Clinical Gerontology, n. 27, p. 3-18, 2004.

JORM, A. F. Is depression a risk factor for dementia or cognitive decline? Gerontology, n. 46, p. 219-227, 2000.

JORM, A. F.; CHRISTENSEN, H.; KORTEN, A. E.; et al. Memory complaints as a precursor of memory impairment in older people: a longitudinal analysis over 7-8 years. Psychological Medicine, n. 31, p. 441-9, 2001. 
KOCKLER, M.; HEUN, R. Gender differences of depressive syntoms in depressed and non depressed elderly persons. International Journal of Geriatric Psychiatry, n. 17, p. 65-72, 2002.

KOEHLER, M.; RABINOWITZ, T.; HIRDES, J. Measuring depression in nursing home residents with the MDS and GDS: an observational psychometric study. BioMed Central Geriatrics, n. 5, p. 1-3, 2005.

LEE, J. S.; POTTER, G. G.; WAGNER, H. R et al. Persistent mild cognitive impairment in geriatric depression. International Psychogeriatrics, v. 19, p. 125-135, 2007.

LIMA, M. S. Epidemiologia e impacto social. Revista Brasileira de Psiquiatria, São Paulo, v. 21, suppl.1, p. 01-05, 1999.

LIMA, M. S.; BÉRIA, J. U.; TOMASI, E. et al. Stressful life events and minor psychiatric disorders: an estimate of the population attributable fraction in a Brazilian community-based study. International Journal of Psychiatry in Medicine (IJPM), n. 26, p. 213-24, 1996.

LYKETSOS, C. G.; STEELE, C.; BAKER, L.; et al. Major and minor depression in Alzheimer's disease: prevalence and impact. Journa of Neuropsychiatry \& Clinical Neuroscience, n. 9, p. 556-61, 1997.

MARI, J. J.; ALMEIDA-FILHO, N.; COUTINHO, E. et al. The epidemiology of psychotropic use in the city of São Paulo. Psychological Medicine, n. 23, p. 467-74, 1993.

MOSSEY, J. M.; GALLAGHER, R. M. The longitudinal occurrence and impact of comorbid chronic pain and chronic depression over two years in continuing care retirement community residents. Pain Medicine, n. 5, p. 335-48, 2004.

NITRINI, R.; CARAMELLI, P.; HERRERA, J. R. E.; et al. Incidence of dementia in a community-dwelling Brazilian population. Alzheimer Disease and Associated Disorders, n. 18, p. 241-246, 2004.
OWNBY, R. L.; CROCCO, E.; ACEVEDO, A.; JOHN, V.; et al. Depression and risk for Alzheimer disease: systematic review, meta-analysis, and metaregression analysis. Archivesof General Psychiatry, n. 63, p. 530538, 2006.

PAULO, D. L. V.; YASSUDA, M. S. Queixas de memória de idosos e sua relação com escolaridade, desempenho cognitivo e sintomas de depressão e ansiedade. Revista de Psiquiatria Clínica, São Paulo, v. 37, n. 1, p. 23-26, 2010.

PERRINO, T.; MASON, C. A.; BROWN, S. C. et al. Longitudinal Relationships Between Cognitive Functioning and Depressive Symptoms Among Hispanic Older Adults. Journal of Gerontology, Serie B, v. 63, n. 5, p. 309-317, 2008.

RABELO, D. F. Comprometimento Cognitivo Leve em Idosos: avaliação, fatores associados e possibilidades de intervenção. Kairós Gerontologia, Campinas, v. 12, n. 2, p. 65-79, 2009.

RAPP, M. A.; DAHLMAN, K.; SANO, M.; et al. Neuropsychological differences between late-onset and recurrent geriatric major depression. American Journal of Psychiatry, n. 162, p. 691-8, 2005.

REISCHIES, F. M.; NEU, P. Comorbidity of mild cognitive disorder and depression - a neuropsychological analysis. European Archives of Psychiatry and Clinical Neuroscience, v. 50, p. 186-193, 2000.

REYS, B. N. et al. Diagnóstico de demência, depressão e psicose em idosos por avaliação Cognitiva breve. Revista da Associação Medica Brasileira, São Paulo, v. 52, n. 6, p. 401-404, 2006.

SACZYNSKI, J. S.; BEISER, A.; SESHADRI, S. et al. Depressive symptoms and risk of dementia. Neurology, v. 75 n. 1, p. 35-41, 2010.

SOARES, E.; COELHO, M. O.; CARVALHO, S. M. R. Capacidade funcional, declínio cognitivo e depressão em idosos institucionali- 
zados: possibilidade de relações e correlações. Kairós Gerontologia, Campinas, v. 15, n. 5, p. 117-139, 2012.

SOARES, E.; DEMARTINI, S. M.; SUZUKI, M. M. et al. Estudo epidemiológico do perfil do idoso institucionalizado em instituições do interior paulista. Revista Ciência em Extensão, São Paulo, v. 8, n. 1, p. 35-59, 2012.

SOARES, E.; OLIVEIRA, T. P; KOMATSU, P. S. et al. Projeto Memória e Envelhecimento: capacitando profissionais e aprimorando aspectos cognitivos em idosos institucionalizados. Revista Brasileira de Ciências do Envelhecimento Humano - RBCEH, Passo Fundo, v. 7, n. 1, p. 62-73, 2010.

SOUSA, R. L.; MEDEIROS, J. G. M.; MOURA, A. C. L. de. et al. Validade e fidedignidade da Escala de Depressão Geriátrica na identificação de idosos deprimidos em um hospital geral. Jornal Brasileiro de Psiquiatria, Rio de Janeiro, v. 56, n. 2, p. 102-107, 2007.

STEWART, J. Depression and cognitive decline - Editorial commentary:Depressive symptoms and cognitive decline - disentangling the effect of affect. Journal of Neurology, Neurosurgery \& Psychiatry, v. 75, p. 5, 2004.

STÜSSI, L. H.; FORTES, L. H. S. D.; RABELO, D. F. Fatores associados ao declínio cognitivo de idosos residentes na comunidade de Patos de Minas-MG. Perquirere, Patos de Minas, v. 7, n. 1, p. 53-62, 2009.

SULLIVAN, M. D.; KATON, W. J.; LOVATO, L. C.; et al. Association of depression with accelerated cognitive decline among patients with type 2 diabetes in the ACCORD-MIND trial. Journal of the American Medical Association - JAMA Psychiatry, v. 70, n. 10, p. 1041-1047, 2013.

SWEATT, J. D. Epigenetics and cognitive aging. Science, v. 328, n. 5979, p. 701-702, 2010.
WANG, L.; Van BELLE, G.; CRANE, P. K.; et al. Subjective memory deterioration and future dementia in people aged 65 and older. Journal of American Geriatrics Society, v. 52, n. 12, p. 2045-2051, 2004.

WANG, P. N.; WANG, S. J.; FUH, J. L.; et al. Subjective memory complaint in relation to cognitive performance and depression: a longitudinal study of a rural Chinese population. Journal of American Geriatrics Society, v. 48, n. 3, p. 295-299, 2000.

WILSON, R. S.; MENDES DE LEON, C. F.; BENNETT, D. A. et al. Depressive symptoms and cognitive decline in a community population of older persons. Journal of Neurology, Neurosurgery \& Psychiatry, n. 75, p. 126-129, 2004.

YESAVAGE J.A.; BRINK, T.L.; ROSE T.L. et al. Development and validation of a geriatric depression screening scale: a preliminary report. Journal of Psychiatric Research, n, 17, v. 1, p. 37-49, 1983.

YOCHIM, B. P.; MACNEILL, S. E.; LICHTENBERG, P. A. "Vascular depression" predicts verbal fluency in older adults. Journalof Clinical and Experimental Neuropsychology, n. 28, p. 495-508, 2006.

XAVIER, F.; FERRAZ, M. P. T.; ARGIMON, I.; et al. A relevância clínica da percepção subjetiva de perda de memória por pessoas de idade. Revista Brasileira de Neurologia, Rio de Janeiro, v. 37, n. 1, p. 24-28, 2001. 